\title{
About Supergraphs. Part II
}

\author{
Sebastian Koch \\ Johannes Gutenberg University \\ Mainz, Germany ${ }^{1}$
}

\begin{abstract}
Summary. In the previous article [5] supergraphs and several specializations to formalize the process of drawing graphs were introduced. In this paper another such operation is formalized in Mizar [1, 2]: drawing a vertex and then immediately drawing edges connecting this vertex with a subset of the other vertices of the graph. In case the new vertex is joined with all vertices of a given graph $G$, this is known as the join of $G$ and the trivial loopless graph $K_{1}$. While the join of two graphs is known and found in standard literature (like [9], 4], 8] and [3]), the operation discribed in this article is not.

Alongside the new operation a mode to reverse the directions of a subset of the edges of a graph is introduced. When all edge directions of a graph are reversed, this is commonly known as the converse of a (directed) graph.
\end{abstract}

MSC: 05C76 03B35 68T99

Keywords: supergraph; graph operations

MML identifier: GLIB_007, version: 8.1.08 5.52.1328

\section{Reversing Edge Directions}

From now on $G, G_{2}$ denote graphs, $V, E$ denote sets, and $v$ denotes an object. Let us consider $G$ and $E$. by

A graph given by reversing directions of the edges $E$ of $G$ is a graph defined

(Def. 1) (i) the vertices of $i t=$ the vertices of $G$ and the edges of $i t=$ the edges of $G$ and the source of $i t=($ the source of $G)+\cdot($ the target of $G)\lceil E$ and the target of $i t=($ the target of $G)+\cdot$ (the source of $G)\lceil E$, if $E \subseteq$ the edges of $G$,

\footnotetext{
${ }^{1}$ mailto: skoch02@students.uni-mainz.de
} 
(ii) it $\approx G$, otherwise.

A graph given by reversing directions of the edges of $G$ is a graph given by reversing directions of the edges of $G$ of $G$. Now we state the propositions:

(1) Let us consider graphs $G_{1}, G_{2}$ given by reversing directions of the edges $E$ of $G$. Then $G_{1} \approx G_{2}$.

(2) Let us consider a graph $G_{1}$ given by reversing directions of the edges $E$ of $G$. Suppose $G_{1} \approx G_{2}$. Then $G_{2}$ is a graph given by reversing directions of the edges $E$ of $G$.

Let us consider $G_{2}, E$, and a graph $G_{1}$ given by reversing directions of the edges $E$ of $G_{2}$. Now we state the propositions:

(3) $G_{2}$ is a graph given by reversing directions of the edges $E$ of $G_{1}$.

(4) (i) the vertices of $G_{1}=$ the vertices of $G_{2}$, and

(ii) the edges of $G_{1}=$ the edges of $G_{2}$.

(5) Let us consider a graph $G_{1}$ given by reversing directions of the edges of $G_{2}$. Then $G_{2}$ is a graph given by reversing directions of the edges of $G_{1}$. The theorem is a consequence of (4) and (3).

(6) Let us consider a trivial graph $G_{2}$, a set $E$, and a graph $G_{1}$. Then $G_{1} \approx G_{2}$ if and only if $G_{1}$ is a graph given by reversing directions of the edges $E$ of $G_{2}$.

Let us consider $G_{2}, E$, a graph $G_{1}$ given by reversing directions of the edges $E$ of $G_{2}$, and objects $v_{1}, e, v_{2}$. Now we state the propositions:

(7) If $E \subseteq$ the edges of $G_{2}$ and $e \in E$, then $e$ joins $v_{1}$ to $v_{2}$ in $G_{2}$ iff $e$ joins $v_{2}$ to $v_{1}$ in $G_{1}$. The theorem is a consequence of (3) and (4).

(8) If $E \subseteq$ the edges of $G_{2}$ and $e \notin E$, then $e$ joins $v_{1}$ to $v_{2}$ in $G_{2}$ iff $e$ joins $v_{1}$ to $v_{2}$ in $G_{1}$. The theorem is a consequence of (3) and (4).

(9) $e$ joins $v_{1}$ and $v_{2}$ in $G_{2}$ if and only if $e$ joins $v_{1}$ and $v_{2}$ in $G_{1}$. The theorem is a consequence of (3).

(10) Let us consider a graph $G_{1}$ given by reversing directions of the edges $E$ of $G_{2}$. Then $v$ is a vertex of $G_{1}$ if and only if $v$ is a vertex of $G_{2}$.

Let us consider $G_{2}, E, V$, and a graph $G_{1}$ given by reversing directions of the edges $E$ of $G_{2}$. Now we state the propositions:

(11) $G_{1}$.edgesBetween $(V)=G_{2}$.edgesBetween $(V)$.

PROOF:

For every object $e, e \in G_{1}$.edgesBetween $(V)$ iff $e \in G_{2}$.edgesBetween $(V)$.

(12) $G_{1}$.edgesInOut $(V)=G_{2}$.edgesInOut $(V)$.

Proof: For every object $e, e \in G_{1}$.edgesInOut $(V)$ iff $e \in G_{2}$.edgesInOut $(V)$. 
(13) Let us consider a graph $G_{1}$ given by reversing directions of the edges $E$ of $G_{2}$, a vertex $v_{1}$ of $G_{1}$, and a vertex $v_{2}$ of $G_{2}$. If $v_{1}=v_{2}$, then $v_{1}$.edgesInOut ()$=v_{2}$.edgesInOut () . The theorem is a consequence of (12).

Let us consider $G_{2}, E$, and a graph $G_{1}$ given by reversing directions of the edges $E$ of $G_{2}$. Now we state the propositions:

(14) Every walk of $G_{2}$ is a walk of $G_{1}$. The theorem is a consequence of (4) and (9).

(15) Every walk of $G_{1}$ is a walk of $G_{2}$. The theorem is a consequence of (3) and (14).

(16) Let us consider a graph $G_{1}$ given by reversing directions of the edges $E$ of $G_{2}$, a walk $W_{2}$ of $G_{2}$, and a walk $W_{1}$ of $G_{1}$. Suppose $E \subseteq$ the edges of $G_{2}$ and $W_{1}=W_{2}$ and $W_{2}$.edges ()$\subseteq E$. Then $W_{1}$ is directed if and only if $W_{2}$.reverse() is directed.

PRoOF: For every odd element $n$ of $\mathbb{N}$ such that $n<$ len $W_{1}$ holds $W_{1}(n+1)$ joins $W_{1}(n)$ to $W_{1}(n+2)$ in $G_{1}$ by [6, (1)], [7, (12)].

(17) Let us consider a graph $G_{1}$ given by reversing directions of the edges of $G_{2}$, a walk $W_{2}$ of $G_{2}$, and a walk $W_{1}$ of $G_{1}$. Suppose $W_{1}=W_{2}$. Then $W_{1}$ is directed if and only if $W_{2}$.reverse() is directed. The theorem is a consequence of (16).

(18) Let us consider a graph $G_{1}$ given by reversing directions of the edges $E$ of $G_{2}$, a walk $W_{2}$ of $G_{2}$, and a walk $W_{1}$ of $G_{1}$. If $W_{1}=W_{2}$, then $W_{1}$ is chordal iff $W_{2}$ is chordal. The theorem is a consequence of (3).

(19) Let us consider a graph $G_{1}$ given by reversing directions of the edges $E$ of $G_{2}$, and objects $v_{1}, v_{2}$. Then there exists a walk $W_{1}$ of $G_{1}$ such that $W_{1}$ is walk from $v_{1}$ to $v_{2}$ if and only if there exists a walk $W_{2}$ of $G_{2}$ such that $W_{2}$ is walk from $v_{1}$ to $v_{2}$. The theorem is a consequence of (15) and (14).

(20) Let us consider a graph $G_{1}$ given by reversing directions of the edges $E$ of $G_{2}$, a vertex $v_{1}$ of $G_{1}$, and a vertex $v_{2}$ of $G_{2}$. If $v_{1}=v_{2}$, then $G_{1}$.reachableFrom $\left(v_{1}\right)=G_{2}$.reachableFrom $\left(v_{2}\right)$. The theorem is a consequence of (19).

(21) Let us consider a graph $G_{1}$ given by reversing directions of the edges $E$ of $G_{2}$. Then

(i) $G_{1} \cdot \operatorname{componentSet}()=G_{2} \cdot \operatorname{componentSet}()$, and

(ii) $G_{1} \cdot \operatorname{numComponents}()=G_{2}$.numComponents().

The theorem is a consequence of (10) and (20).

Let $G$ be a trivial graph and $E$ be a set. Observe that every graph given by reversing directions of the edges $E$ of $G$ is trivial. 
Let $G$ be a non trivial graph. Let us observe that every graph given by reversing directions of the edges $E$ of $G$ is non trivial.

Now we state the propositions:

(22) Let us consider a graph $G_{1}$ given by reversing directions of the edges $E$ of $G_{2}$, a set $v$, and a subgraph $G_{3}$ of $G_{1}$ with vertex $v$ removed. Then every subgraph of $G_{2}$ with vertex $v$ removed is a graph given by reversing directions of the edges $E \backslash G_{1}$.edgesInOut $(\{v\})$ of $G_{3}$. The theorem is a consequence of (11), (2), (3), and (6).

(23) Let us consider a graph $G_{1}$ given by reversing directions of the edges $E$ of $G_{2}$, a vertex $v_{1}$ of $G_{1}$, and a vertex $v_{2}$ of $G_{2}$. Suppose $v_{1}=v_{2}$. Then

(i) $v_{1}$ is isolated iff $v_{2}$ is isolated, and

(ii) $v_{1}$ is endvertex iff $v_{2}$ is endvertex, and

(iii) $v_{1}$ is cut-vertex iff $v_{2}$ is cut-vertex.

The theorem is a consequence of (3).

Let us consider $G_{2}, E$, and a graph $G_{1}$ given by reversing directions of the edges $E$ of $G_{2}$. Now we state the propositions:

(i) $G_{1} \cdot \operatorname{order}()=G_{2}$.order(), and

(ii) $G_{1} \cdot \operatorname{size}()=G_{2} \cdot \operatorname{size}()$.

The theorem is a consequence of (4).

(25) Suppose $E \subseteq$ the edges of $G_{2}$ and $G_{2}$ is non-directed-multi and for every objects $e_{1}, e_{2}, v_{1}, v_{2}$ such that $e_{1}$ joins $v_{1}$ and $v_{2}$ in $G_{2}$ and $e_{2}$ joins $v_{1}$ and $v_{2}$ in $G_{2}$ holds $e_{1}, e_{2} \in E$ or $e_{1} \notin E$ and $e_{2} \notin E$. Then $G_{1}$ is non-directedmulti.

Proof: For every objects $e_{1}, e_{2}, v_{1}, v_{2}$ such that $e_{1}$ joins $v_{1}$ to $v_{2}$ in $G_{1}$ and $e_{2}$ joins $v_{1}$ to $v_{2}$ in $G_{1}$ holds $e_{1}=e_{2}$.

Let $G$ be a non-directed-multi graph. Let us note that every graph given by reversing directions of the edges of $G$ is non-directed-multi.

Let $G$ be a non non-directed-multi graph. Observe that every graph given by reversing directions of the edges of $G$ is non non-directed-multi.

Let $G$ be a non-multi graph and $E$ be a set. One can verify that every graph given by reversing directions of the edges $E$ of $G$ is non-multi.

Let $G$ be a non non-multi graph. Let us note that every graph given by reversing directions of the edges $E$ of $G$ is non non-multi.

Let $G$ be a loopless graph. One can check that every graph given by reversing directions of the edges $E$ of $G$ is loopless.

Let $G$ be a non loopless graph. One can check that every graph given by reversing directions of the edges $E$ of $G$ is non loopless. 
Let $G$ be a connected graph. Let us observe that every graph given by reversing directions of the edges $E$ of $G$ is connected.

Let $G$ be a non connected graph. Observe that every graph given by reversing directions of the edges $E$ of $G$ is non connected.

Let $G$ be an acyclic graph. Note that every graph given by reversing directions of the edges $E$ of $G$ is acyclic.

Let $G$ be a non acyclic graph. One can verify that every graph given by reversing directions of the edges $E$ of $G$ is non acyclic.

Let $G$ be a complete graph. Observe that every graph given by reversing directions of the edges $E$ of $G$ is complete.

Let $G$ be a non complete graph. Observe that every graph given by reversing directions of the edges $E$ of $G$ is non complete.

Let $G$ be a chordal graph. Note that every graph given by reversing directions of the edges $E$ of $G$ is chordal.

Let $G$ be a finite graph. Let us note that every graph given by reversing directions of the edges $E$ of $G$ is finite.

Let $G$ be a non finite graph. One can verify that every graph given by reversing directions of the edges $E$ of $G$ is non finite.

Now we state the propositions:

(26) Let us consider a graph $G_{1}$ given by reversing directions of the edges of $G_{2}$. Then

(i) the source of $G_{1}=$ the target of $G_{2}$, and

(ii) the target of $G_{1}=$ the source of $G_{2}$.

(27) Let us consider a graph $G_{1}$ given by reversing directions of the edges of $G_{2}$, and objects $v_{1}, e, v_{2}$. Then $e$ joins $v_{1}$ to $v_{2}$ in $G_{2}$ if and only if $e$ joins $v_{2}$ to $v_{1}$ in $G_{1}$. The theorem is a consequence of (26).

\section{Adding a Vertex and Several Edges to a Graph}

Let us consider $G, v$, and $V$.

A supergraph of $G$ extended by vertex $v$ and edges from $v$ to $V$ of $G$ is a supergraph of $G$ defined by

(Def. 2) (i) the vertices of $i t=$ (the vertices of $G) \cup\{v\}$ and the edges of it $=$ (the edges of $G) \cup(V \longmapsto$ (the edges of $G)$ ) and the source of $i t=$ (the source of $G)+\cdot((V \longmapsto$ (the edges of $G)) \longmapsto v)$ and the target of $i t=($ the target of $G)+\cdot \pi_{1}(V \otimes\{$ the edges of $G\})$, if $V \subseteq$ the vertices of $G$ and $v \notin$ the vertices of $G$,

(ii) it $\approx G$, otherwise. 
A supergraph of $G$ extended by vertex $v$ and edges from $V$ of $G$ to $v$ is a supergraph of $G$ defined by

(Def. 3) (i) the vertices of it $=$ (the vertices of $G) \cup\{v\}$ and the edges of it $=($ the edges of $G) \cup(V \longmapsto($ the edges of $G))$ and the source of $i t=($ the source of $G)+\cdot \pi_{1}(V \otimes\{$ the edges of $G\})$ and the target of $i t=$ (the target of $G)+\cdot((V \longmapsto$ (the edges of $G)) \longmapsto v$ ), if $V \subseteq$ the vertices of $G$ and $v \notin$ the vertices of $G$,

(ii) it $\approx G$, otherwise.

A supergraph of $G$ extended by vertex $v$ and edges from $v$ to the vertices of $G$ is a supergraph of $G$ extended by vertex $v$ and edges from $v$ to the vertices of $G$ of $G$.

A supergraph of $G$ extended by vertex $v$ and edges from the vertices of $G$ to $v$ is a supergraph of $G$ extended by vertex $v$ and edges from the vertices of $G$ of $G$ to $v$. Now we state the propositions:

(28) Let us consider supergraphs $G_{1}, G_{2}$ of $G$ extended by vertex $v$ and edges from $v$ to $V$ of $G$. Then $G_{1} \approx G_{2}$.

(29) Let us consider supergraphs $G_{1}, G_{2}$ of $G$ extended by vertex $v$ and edges from $V$ of $G$ to $v$. Then $G_{1} \approx G_{2}$.

(30) Let us consider a supergraph $G_{1}$ of $G$ extended by vertex $v$ and edges from $v$ to $V$ of $G$. Suppose $G_{1} \approx G_{2}$. Then $G_{2}$ is a supergraph of $G$ extended by vertex $v$ and edges from $v$ to $V$ of $G$.

(31) Let us consider a supergraph $G_{1}$ of $G$ extended by vertex $v$ and edges from $V$ of $G$ to $v$. Suppose $G_{1} \approx G_{2}$. Then $G_{2}$ is a supergraph of $G$ extended by vertex $v$ and edges from $V$ of $G$ to $v$.

(32) Let us consider a supergraph $G_{1}$ of $G$ extended by vertex $v$ and edges from $v$ to $V$ of $G$, and a supergraph $G_{2}$ of $G$ extended by vertex $v$ and edges from $V$ of $G$ to $v$. Then

(i) the vertices of $G_{1}=$ the vertices of $G_{2}$, and

(ii) the edges of $G_{1}=$ the edges of $G_{2}$.

(33) Let us consider a supergraph $G_{1}$ of $G_{2}$ extended by vertex $v$ and edges from $v$ to $V$ of $G_{2}$. Suppose $V \subseteq$ the vertices of $G_{2}$ and $v \notin$ the vertices of $G_{2}$. Then $G_{1}$.edgesOutOf $(\{v\})=V \longmapsto\left(\right.$ the edges of $\left.G_{2}\right)$.

Proof: For every object $e, e \in G_{1}$.edgesOutOf $(\{v\})$ iff $e \in V \longmapsto$ (the edges of $G_{2}$ ).

(34) Let us consider a supergraph $G_{1}$ of $G_{2}$ extended by vertex $v$ and edges from $V$ of $G_{2}$ to $v$. Suppose $V \subseteq$ the vertices of $G_{2}$ and $v \notin$ the vertices of $G_{2}$. Then $G_{1}$.edgesInto $(\{v\})=V \longmapsto\left(\right.$ the edges of $\left.G_{2}\right)$. 
Proof: For every object $e, e \in G_{1}$.edgesInto $(\{v\})$ iff $e \in V \longmapsto$ (the edges of $G_{2}$ ).

(35) Let us consider a supergraph $G_{1}$ of $G$ extended by vertex $v$ and edges from $v$ to $V$ of $G$, and a supergraph $G_{2}$ of $G$ extended by vertex $v$ and edges from $V$ of $G$ to $v$. Suppose $V \subseteq$ the vertices of $G$ and $v \notin$ the vertices of $G$. Then

(i) $G_{2}$ is a graph given by reversing directions of the edges $G_{1}$.edgesOutOf $(\{v\})$ of $G_{1}$, and

(ii) $G_{1}$ is a graph given by reversing directions of the edges $G_{2}$.edgesInto $(\{v\})$ of $G_{2}$.

The theorem is a consequence of (33) and (34).

(36) Let us consider a supergraph $G_{1}$ of $G$ extended by vertex $v$ and edges from $v$ to $V$ of $G$, a supergraph $G_{2}$ of $G$ extended by vertex $v$ and edges from $V$ of $G$ to $v$, and objects $v_{1}, e, v_{2}$. Then $e$ joins $v_{1}$ and $v_{2}$ in $G_{1}$ if and only if $e$ joins $v_{1}$ and $v_{2}$ in $G_{2}$. The theorem is a consequence of (35) and (9).

(37) Let us consider a supergraph $G_{1}$ of $G$ extended by vertex $v$ and edges from $v$ to $V$ of $G$, a supergraph $G_{2}$ of $G$ extended by vertex $v$ and edges from $V$ of $G$ to $v$, and an object $w$. Then $w$ is a vertex of $G_{1}$ if and only if $w$ is a vertex of $G_{2}$.

(38) Let us consider a supergraph $G_{1}$ of $G_{2}$ extended by vertex $v$ and edges from $v$ to $V$ of $G_{2}$. Suppose $V \subseteq$ the vertices of $G_{2}$ and $v \notin$ the vertices of $G_{2}$. Let us consider objects $e_{1}, u$. Then

(i) $e_{1}$ does not join $u$ to $v$ in $G_{1}$, and

(ii) if $u \notin V$, then $e_{1}$ does not join $v$ to $u$ in $G_{1}$, and

(iii) for every object $e_{2}$ such that $e_{1}$ joins $v$ to $u$ in $G_{1}$ and $e_{2}$ joins $v$ to $u$ in $G_{1}$ holds $e_{1}=e_{2}$.

Proof: $e_{1}$ does not join $u$ to $v$ in $G_{1}$. If $u \notin V$, then $e_{1}$ does not join $v$ to $u$ in $G_{1} . e_{1} \notin$ the edges of $G_{2}$ and $e_{2} \notin$ the edges of $G_{2}$. Consider $x_{1}, y_{1}$ being objects such that $x_{1} \in V$ and $y_{1} \in$ the edges of $\left.G_{2}\right\}$ and $e_{1}=\left\langle x_{1}\right.$, $\left.y_{1}\right\rangle$. Consider $x_{2}, y_{2}$ being objects such that $x_{2} \in V$ and $y_{2} \in\{$ the edges of $\left.G_{2}\right\}$ and $e_{2}=\left\langle x_{2}, y_{2}\right\rangle$.

(39) Let us consider a supergraph $G_{1}$ of $G_{2}$ extended by vertex $v$ and edges from $V$ of $G_{2}$ to $v$. Suppose $V \subseteq$ the vertices of $G_{2}$ and $v \notin$ the vertices of $G_{2}$. Let us consider objects $e_{1}, u$. Then

(i) $e_{1}$ does not join $v$ to $u$ in $G_{1}$, and

(ii) if $u \notin V$, then $e_{1}$ does not join $u$ to $v$ in $G_{1}$, and 
(iii) for every object $e_{2}$ such that $e_{1}$ joins $u$ to $v$ in $G_{1}$ and $e_{2}$ joins $u$ to $v$ in $G_{1}$ holds $e_{1}=e_{2}$.

Proof: $e_{1}$ does not join $v$ to $u$ in $G_{1}$. If $u \notin V$, then $e_{1}$ does not join $u$ to $v$ in $G_{1} . e_{1} \notin$ the edges of $G_{2}$ and $e_{2} \notin$ the edges of $G_{2}$. Consider $x_{1}, y_{1}$ being objects such that $x_{1} \in V$ and $y_{1} \in$ the edges of $\left.G_{2}\right\}$ and $e_{1}=\left\langle x_{1}\right.$, $\left.y_{1}\right\rangle$. Consider $x_{2}, y_{2}$ being objects such that $x_{2} \in V$ and $y_{2} \in$ the edges of $\left.G_{2}\right\}$ and $e_{2}=\left\langle x_{2}, y_{2}\right\rangle$.

(40) Let us consider a supergraph $G_{1}$ of $G_{2}$ extended by vertex $v$ and edges from $v$ to $V$ of $G_{2}$. Suppose $V \subseteq$ the vertices of $G_{2}$ and $v \notin$ the vertices of $G_{2}$. Let us consider objects $e, v_{1}, v_{2}$. Suppose $v_{1} \neq v$. If $e$ joins $v_{1}$ to $v_{2}$ in $G_{1}$, then $e$ joins $v_{1}$ to $v_{2}$ in $G_{2}$.

Proof: $e \in$ the edges of $G_{2}$.

(41) Let us consider a supergraph $G_{1}$ of $G_{2}$ extended by vertex $v$ and edges from $V$ of $G_{2}$ to $v$. Suppose $V \subseteq$ the vertices of $G_{2}$ and $v \notin$ the vertices of $G_{2}$. Let us consider objects $e, v_{1}, v_{2}$. Suppose $v_{2} \neq v$. If $e$ joins $v_{1}$ to $v_{2}$ in $G_{1}$, then $e$ joins $v_{1}$ to $v_{2}$ in $G_{2}$.

Proof: $e \in$ the edges of $G_{2}$.

(42) Let us consider a supergraph $G_{1}$ of $G_{2}$ extended by vertex $v$ and edges from $v$ to $V$ of $G_{2}$, and an object $v_{1}$. Suppose $V \subseteq$ the vertices of $G_{2}$ and $v \notin$ the vertices of $G_{2}$ and $v_{1} \in V$. Then $\left\langle v_{1}\right.$, the edges of $\left.G_{2}\right\rangle$ joins $v$ to $v_{1}$ in $G_{1}$.

(43) Let us consider a supergraph $G_{1}$ of $G_{2}$ extended by vertex $v$ and edges from $V$ of $G_{2}$ to $v$, and an object $v_{1}$. Suppose $V \subseteq$ the vertices of $G_{2}$ and $v \notin$ the vertices of $G_{2}$ and $v_{1} \in V$. Then $\left\langle v_{1}\right.$, the edges of $\left.G_{2}\right\rangle$ joins $v_{1}$ to $v$ in $G_{1}$.

Let us consider $G, v, V$, a supergraph $G_{1}$ of $G$ extended by vertex $v$ and edges from $v$ to $V$ of $G$, and a supergraph $G_{2}$ of $G$ extended by vertex $v$ and edges from $V$ of $G$ to $v$. Now we state the propositions:

(44) Every walk of $G_{1}$ is a walk of $G_{2}$. The theorem is a consequence of (35) and (14).

(45) Every walk of $G_{2}$ is a walk of $G_{1}$. The theorem is a consequence of (35) and (14).

Let us consider $G, v$, and $V$.

A supergraph of $G$ extended by vertex $v$ and edges between $v$ and $V$ of $G$ is a supergraph of $G$ defined by

(Def. 4) (i) the vertices of $i t=($ the vertices of $G) \cup\{v\}$ and for every object $e$, $e$ does not join $v$ and $v$ in it and for every object $v_{1}$, if $v_{1} \notin V$, then $e$ does not join $v_{1}$ and $v$ in $i t$ and for every object $v_{2}$ such that $v_{1} \neq v$ 
and $v_{2} \neq v$ and $e$ joins $v_{1}$ to $v_{2}$ in it holds $e$ joins $v_{1}$ to $v_{2}$ in $G$ and there exists a set $E$ such that $\overline{\bar{V}}=\overline{\bar{E}}$ and $E$ misses the edges of $G$ and the edges of $i t=($ the edges of $G) \cup E$ and for every object $v_{1}$ such that $v_{1} \in V$ there exists an object $e_{1}$ such that $e_{1} \in E$ and $e_{1}$ joins $v_{1}$ and $v$ in it and for every object $e_{2}$ such that $e_{2}$ joins $v_{1}$ and $v$ in $i t$ holds $e_{1}=e_{2}$, if $V \subseteq$ the vertices of $G$ and $v \notin$ the vertices of $G$,

(ii) it $\approx G$, otherwise.

A supergraph of $G$ extended by vertex $v$ and edges between $v$ and the vertices of $G$ is a supergraph of $G$ extended by vertex $v$ and edges between $v$ and the vertices of $G$ of $G$.

One can verify that a supergraph of $G$ extended by vertex $v$ and edges from $v$ to $V$ of $G$ is a supergraph of $G$ extended by vertex $v$ and edges between $v$ and $V$ of $G$.

Note that a supergraph of $G$ extended by vertex $v$ and edges from $V$ of $G$ to $v$ is a supergraph of $G$ extended by vertex $v$ and edges between $v$ and $V$ of $G$. Now we state the propositions:

(46) Let us consider a supergraph $G_{1}$ of $G_{2}$ extended by vertex $v$ and edges between $v$ and $\emptyset$ of $G_{2}$. Then the edges of $G_{2}=$ the edges of $G_{1}$.

(47) Let us consider a non empty set $V$, and a supergraph $G_{1}$ of $G_{2}$ extended by vertex $v$ and edges between $v$ and $V$ of $G_{2}$. Suppose $V \subseteq$ the vertices of $G_{2}$ and $v \notin$ the vertices of $G_{2}$. Then the edges of $G_{1} \neq \emptyset$.

(48) Let us consider a supergraph $G_{1}$ of $G$ extended by vertex $v$ and edges between $v$ and $V$ of $G$. Suppose $G_{1} \approx G_{2}$. Then $G_{2}$ is a supergraph of $G$ extended by vertex $v$ and edges between $v$ and $V$ of $G$.

(49) Let us consider a supergraph $G_{1}$ of $G_{2}$ extended by vertex $v$ and edges between $v$ and $V$ of $G_{2}$, and objects $v_{1}, e, v_{2}$. Suppose $V \subseteq$ the vertices of $G_{2}$ and $v \notin$ the vertices of $G_{2}$ and $v_{1} \neq v$ and $v_{2} \neq v$ and $e$ joins $v_{1}$ and $v_{2}$ in $G_{1}$. Then $e$ joins $v_{1}$ and $v_{2}$ in $G_{2}$.

(50) Let us consider a supergraph $G_{1}$ of $G_{2}$ extended by vertex $v$ and edges between $v$ and $V$ of $G_{2}$. Suppose $V \subseteq$ the vertices of $G_{2}$ and $v \notin$ the vertices of $G_{2}$. Then $v$ is a vertex of $G_{1}$.

(51) Let us consider a supergraph $G_{1}$ of $G_{2}$ extended by vertex $v$ and edges between $v$ and $V$ of $G_{2}$, a set $E$, and objects $v_{1}, e, v_{2}$. Suppose $V \subseteq$ the vertices of $G_{2}$ and $v \notin$ the vertices of $G_{2}$ and the edges of $G_{1}=$ (the edges of $\left.G_{2}\right) \cup E$ and $E$ misses the edges of $G_{2}$ and $e$ joins $v_{1}$ and $v_{2}$ in $G_{1}$ and $e \notin$ the edges of $G_{2}$. Then

(i) $e \in E$, and 
(ii) $v_{1}=v$ and $v_{2} \in V$ or $v_{2}=v$ and $v_{1} \in V$.

(52) Let us consider a supergraph $G_{1}$ of $G_{2}$ extended by vertex $v$ and edges between $v$ and $V$ of $G_{2}$, and a set $E$. Suppose $V \subseteq$ the vertices of $G_{2}$ and $v \notin$ the vertices of $G_{2}$ and the edges of $G_{1}=$ (the edges of $\left.G_{2}\right) \cup E$ and $E$ misses the edges of $G_{2}$. Then there exist functions $f, g$ from $E$ into $V \cup\{v\}$ such that

(i) the source of $G_{1}=\left(\right.$ the source of $\left.G_{2}\right)+\cdot f$, and

(ii) the target of $G_{1}=\left(\right.$ the target of $\left.G_{2}\right)+\cdot g$, and

(iii) for every object $e$ such that $e \in E$ holds $e$ joins $f(e)$ to $g(e)$ in $G_{1}$ and $(f(e)=v$ iff $g(e) \neq v)$.

Proof: Consider $E_{1}$ being a set such that $\overline{\bar{V}}=\overline{\overline{E_{1}}}$ and $E_{1}$ misses the edges of $G_{2}$ and the edges of $G_{1}=$ (the edges of $\left.G_{2}\right) \cup E_{1}$ and for every object $v_{1}$ such that $v_{1} \in V$ there exists an object $e_{1}$ such that $e_{1} \in E_{1}$ and $e_{1}$ joins $v_{1}$ and $v$ in $G_{1}$ and for every object $e_{2}$ such that $e_{2}$ joins $v_{1}$ and $v$ in $G_{1}$ holds $e_{1}=e_{2}$. Define $\mathcal{P}$ [object, object] $\equiv$ there exists an object $v_{2}$ such that $\$_{1}$ joins $\$_{2}$ to $v_{2}$ in $G_{1}$. For every object $e$ such that $e \in E$ there exists an object $v_{1}$ such that $v_{1} \in V \cup\{v\}$ and $\mathcal{P}\left[e, v_{1}\right]$.

Consider $f$ being a function from $E$ into $V \cup\{v\}$ such that for every object $e$ such that $e \in E$ holds $\mathcal{P}[e, f(e)]$. Define $\mathcal{Q}$ [object, object] $\equiv \$_{1}$ joins $f\left(\$_{1}\right)$ to $\$_{2}$ in $G_{1}$. For every object $e$ such that $e \in E$ there exists an object $v_{2}$ such that $v_{2} \in V \cup\{v\}$ and $\mathcal{Q}\left[e, v_{2}\right]$.

Consider $g$ being a function from $E$ into $V \cup\{v\}$ such that for every object $e$ such that $e \in E$ holds $\mathcal{Q}[e, g(e)]$. For every object $e$ such that $e \in \operatorname{dom}\left(\right.$ the source of $\left.G_{1}\right)$ holds (the source of $\left.G_{1}\right)(e)=(($ the source of $\left.\left.G_{2}\right)+\cdot f\right)(e)$. For every object $e$ such that $e \in \operatorname{dom}\left(\right.$ the target of $\left.G_{1}\right)$ holds (the target of $\left.G_{1}\right)(e)=\left(\left(\right.\right.$ the target of $\left.\left.G_{2}\right)+\cdot g\right)(e)$.

(53) Let us consider a supergraph $G_{1}$ of $G_{2}$ extended by vertex $v$ and edges between $v$ and $V$ of $G_{2}$. Suppose $V \subseteq$ the vertices of $G_{2}$ and $v \notin$ the vertices of $G_{2}$. Then the edges of $G_{2}=G_{1}$.edgesBetween(the vertices of $G_{2}$ ).

Proof: Set $B=G_{1}$.edgesBetween(the vertices of $G_{2}$ ). For every object $e$, $e \in$ the edges of $G_{2}$ iff $e \in B$.

(54) Let us consider a graph $G_{2}$, sets $v, V$, and a supergraph $G_{1}$ of $G_{2}$ extended by vertex $v$ and edges between $v$ and $V$ of $G_{2}$. Suppose $V \subseteq$ the vertices of $G_{2}$ and $v \notin$ the vertices of $G_{2}$. Then $G_{2}$ is a subgraph of $G_{1}$ with vertex $v$ removed. The theorem is a consequence of (53).

(55) Every supergraph of $G_{2}$ extended by vertex $v$ and edges between $v$ and $\emptyset$ of $G_{2}$ is a supergraph of $G_{2}$ extended by $v$. The theorem is a consequence of $(46)$. 
(56) Let us consider an object $v_{1}$, and a supergraph $G_{1}$ of $G_{2}$ extended by vertex $v$ and edges between $v$ and $\left\{v_{1}\right\}$ of $G_{2}$. Suppose $v_{1} \in$ the vertices of $G_{2}$ and $v \notin$ the vertices of $G_{2}$. Then there exists an object $e$ such that

(i) $e \notin$ the edges of $G_{2}$, and

(ii) $G_{1}$ is supergraph of $G_{2}$ extended by vertices $v, v_{1}$ and $e$ between them or supergraph of $G_{2}$ extended by vertices $v_{1}, v$ and $e$ between them.

The theorem is a consequence of (52).

(57) Let us consider a subset $W$ of $V$, and a supergraph $G_{1}$ of $G_{2}$ extended by vertex $v$ and edges between $v$ and $V$ of $G_{2}$. Suppose $V \subseteq$ the vertices of $G_{2}$ and $v \notin$ the vertices of $G_{2}$. Then there exists a function $f$ from $W$ into $G_{1}$.edgesBetween $(W,\{v\})$ such that

(i) $f$ is one-to-one and onto, and

(ii) for every object $w$ such that $w \in W$ holds $f(w)$ joins $w$ and $v$ in $G_{1}$. Proof: Consider $E$ being a set such that $\overline{\bar{V}}=\overline{\bar{E}}$ and $E$ misses the edges of $G_{2}$ and the edges of $G_{1}=$ (the edges of $\left.G_{2}\right) \cup E$ and for every object $v_{1}$ such that $v_{1} \in V$ there exists an object $e_{1}$ such that $e_{1} \in E$ and $e_{1}$ joins $v_{1}$ and $v$ in $G_{1}$ and for every object $e_{2}$ such that $e_{2}$ joins $v_{1}$ and $v$ in $G_{1}$ holds $e_{1}=e_{2}$. Define $\mathcal{P}$ [object, object] $\equiv \$_{2}$ joins $\$_{1}$ and $v$ in $G_{1}$. For every object $w$ such that $w \in W$ there exists an object $e$ such that $e \in G_{1}$.edgesBetween $(W,\{v\})$ and $\mathcal{P}[w, e]$.

Consider $f$ being a function from $W$ into $G_{1}$.edgesBetween $(W,\{v\})$ such that for every object $w$ such that $w \in W$ holds $\mathcal{P}[w, f(w)]$. For every objects $w_{1}, w_{2}$ such that $w_{1}, w_{2} \in W$ and $f\left(w_{1}\right)=f\left(w_{2}\right)$ holds $w_{1}=w_{2}$. For every object $e$ such that $e \in G_{1}$. $\operatorname{edgesBetween}(W,\{v\})$ holds $e \in \operatorname{rng} f$.

(58) Let us consider a supergraph $G_{1}$ of $G_{2}$ extended by vertex $v$ and edges between $v$ and $V$ of $G_{2}$. Suppose $V \subseteq$ the vertices of $G_{2}$ and $v \notin$ the vertices of $G_{2}$ and $E$ misses the edges of $G_{2}$ and the edges of $G_{1}=$ (the edges of $\left.G_{2}\right) \cup E$. Then $E=G_{1}$.edgesBetween $(V,\{v\})$.

Proof: Consider $E_{1}$ being a set such that $\overline{\bar{V}}=\overline{\overline{E_{1}}}$ and $E_{1}$ misses the edges of $G_{2}$ and the edges of $G_{1}=$ (the edges of $\left.G_{2}\right) \cup E_{1}$ and for every object $v_{1}$ such that $v_{1} \in V$ there exists an object $e_{1}$ such that $e_{1} \in E_{1}$ and $e_{1}$ joins $v_{1}$ and $v$ in $G_{1}$ and for every object $e_{2}$ such that $e_{2}$ joins $v_{1}$ and $v$ in $G_{1}$ holds $e_{1}=e_{2}$. For every object $e, e \in E$ iff $e \in G_{1}$.edgesBetween $(V,\{v\})$.

(59) Let us consider a supergraph $G_{1}$ of $G_{2}$ extended by vertex $v$ and edges between $v$ and $V$ of $G_{2}$. Suppose $V \subseteq$ the vertices of $G_{2}$ and $v \notin$ the vertices 
of $G_{2}$. Then

(i) $G_{1}$.edgesBetween $(V,\{v\})$ misses the edges of $G_{2}$, and

(ii) the edges of $G_{1}=\left(\right.$ the edges of $\left.G_{2}\right) \cup G_{1}$.edgesBetween $(V,\{v\})$.

Proof: $G_{1}$.edgesBetween $(V,\{v\}) \cap\left(\right.$ the edges of $\left.G_{2}\right)=\emptyset$. For every object $e$ such that $e \in$ the edges of $G_{1}$ holds $e \in$ (the edges of $\left.G_{2}\right) \cup$ $G_{1}$.edgesBetween $(V,\{v\})$.

(60) Let us consider a graph $G_{3}$, an object $v$, sets $V_{1}, V_{2}$, a supergraph $G_{1}$ of $G_{3}$ extended by vertex $v$ and edges between $v$ and $V_{1} \cup V_{2}$ of $G_{3}$, and a subgraph $G_{2}$ of $G_{1}$ with edges $G_{1}$.edgesBetween $\left(V_{2},\{v\}\right)$ removed. Suppose $V_{1} \cup V_{2} \subseteq$ the vertices of $G_{3}$ and $v \notin$ the vertices of $G_{3}$ and $V_{1}$ misses $V_{2}$. Then $G_{2}$ is a supergraph of $G_{3}$ extended by vertex $v$ and edges between $v$ and $V_{1}$ of $G_{3}$.

Proof: Consider $E$ being a set such that $\overline{\overline{V_{1} \cup V_{2}}}=\overline{\bar{E}}$ and $E$ misses the edges of $G_{3}$ and the edges of $G_{1}=$ (the edges of $\left.G_{3}\right) \cup E$ and for every object $v_{1}$ such that $v_{1} \in V_{1} \cup V_{2}$ there exists an object $e_{1}$ such that $e_{1} \in E$ and $e_{1}$ joins $v_{1}$ and $v$ in $G_{1}$ and for every object $e_{2}$ such that $e_{2}$ joins $v_{1}$ and $v$ in $G_{1}$ holds $e_{1}=e_{2} . E=G_{1}$. edgesBetween $\left(V_{1} \cup V_{2},\{v\}\right)$. For every object $e$ such that $e \in$ the edges of $G_{3}$ holds $e \in$ (the edges of $\left.G_{3}\right) \backslash G_{1}$.edgesBetween $\left(V_{2},\{v\}\right) . G_{2}$ is a supergraph of $G_{3}$.

(61) Let us consider a graph $G_{3}$, an object $v$, a set $V$, a vertex $v_{1}$ of $G_{3}$, and a supergraph $G_{1}$ of $G_{3}$ extended by vertex $v$ and edges between $v$ and $V \cup\left\{v_{1}\right\}$ of $G_{3}$. Suppose $V \subseteq$ the vertices of $G_{3}$ and $v \notin$ the vertices of $G_{3}$ and $v_{1} \notin V$.

Then there exists a supergraph $G_{2}$ of $G_{3}$ extended by vertex $v$ and edges between $v$ and $V$ of $G_{3}$ and there exists an object $e$ such that $e \notin$ the edges of $G_{3}$ and $G_{1}$ is supergraph of $G_{2}$ extended by $e$ between vertices $v$ and $v_{1}$ or supergraph of $G_{2}$ extended by $e$ between vertices $v_{1}$ and $v$.

Proof: Reconsider $W=\left\{v_{1}\right\}$ as a subset of $V \cup\left\{v_{1}\right\}$. Consider $f$ being a function from $W$ into $G_{1}$.edgesBetween $(W,\{v\})$ such that $f$ is one-toone and onto and for every object $w$ such that $w \in W$ holds $f(w)$ joins $w$ and $v$ in $G_{1} \cdot f\left(v_{1}\right) \notin$ the edges of $G_{3} \cdot v$ is a vertex of $G_{1}$.

(62) Let us consider a graph $G_{3}$, an object $v$, a set $V$, a vertex $v_{1}$ of $G_{3}$, an object $e$, and a supergraph $G_{2}$ of $G_{3}$ extended by vertex $v$ and edges between $v$ and $V$ of $G_{3}$. Suppose $V \subseteq$ the vertices of $G_{3}$ and $v \notin$ the vertices of $G_{3}$ and $v_{1} \notin V$ and $e \notin$ the edges of $G_{2}$.

Let us consider a graph $G_{1}$. Suppose $G_{1}$ is supergraph of $G_{2}$ extended by $e$ between vertices $v_{1}$ and $v$ or supergraph of $G_{2}$ extended by $e$ between 
vertices $v$ and $v_{1}$. Then $G_{1}$ is a supergraph of $G_{3}$ extended by vertex $v$ and edges between $v$ and $V \cup\left\{v_{1}\right\}$ of $G_{3}$.

Proof: Consider $E$ being a set such that $\overline{\bar{V}}=\overline{\bar{E}}$ and $E$ misses the edges of $G_{3}$ and the edges of $G_{2}=$ (the edges of $\left.G_{3}\right) \cup E$ and for every object $v_{1}$ such that $v_{1} \in V$ there exists an object $e_{1}$ such that $e_{1} \in E$ and $e_{1}$ joins $v_{1}$ and $v$ in $G_{2}$ and for every object $e_{2}$ such that $e_{2}$ joins $v_{1}$ and $v$ in $G_{2}$ holds $e_{1}=e_{2}$. Consider $f$ being a function such that $f$ is one-to-one and $\operatorname{dom} f=E$ and $\operatorname{rng} f=V$. Set $f_{1}=f+\cdot\left(e \longmapsto v_{1}\right) \cdot \operatorname{rng} f \cap \operatorname{rng}\left(e \longmapsto v_{1}\right)=\emptyset$. For every object $w$ such that $w \in \operatorname{rng} f \cup \operatorname{rng}\left(e \longmapsto v_{1}\right)$ holds $w \in \operatorname{rng} f_{1} \cdot v$ is a vertex of $G_{2}$ and $v_{1}$ is a vertex of $G_{3}$.

Let us consider $G_{2}, v, V$, a supergraph $G_{1}$ of $G_{2}$ extended by vertex $v$ and edges between $v$ and $V$ of $G_{2}$, and a walk $W$ of $G_{1}$. Now we state the propositions:

(63) Suppose $V \subseteq$ the vertices of $G_{2}$ and $v \notin$ the vertices of $G_{2}$. Then

(i) if $W$.edges ()$\subseteq$ the edges of $G_{2}$ and $W$ is not trivial, then $v \notin$ $W$.vertices(), and

(ii) if $v \notin W$.vertices(), then $W$.edges( $) \subseteq$ the edges of $G_{2}$.

Proof: Consider $E$ being a set such that $\overline{\bar{V}}=\overline{\bar{E}}$ and $E$ misses the edges of $G_{2}$ and the edges of $G_{1}=$ (the edges of $\left.G_{2}\right) \cup E$ and for every object $v_{1}$ such that $v_{1} \in V$ there exists an object $e_{1}$ such that $e_{1} \in E$ and $e_{1}$ joins $v_{1}$ and $v$ in $G_{1}$ and for every object $e_{2}$ such that $e_{2}$ joins $v_{1}$ and $v$ in $G_{1}$ holds $e_{1}=e_{2}$. For every object $e$ such that $e \in W$.edges() holds $e \in$ the edges of $G_{2}$.

(64) Suppose $V \subseteq$ the vertices of $G_{2}$ and $v \notin$ the vertices of $G_{2}$ and ( $W$.edges() $\subseteq$ the edges of $G_{2}$ and $W$ is not trivial or $v \notin W$.vertices()). Then $W$ is a walk of $G_{2}$. The theorem is a consequence of (63).

(65) If $W$.vertices ()$\subseteq$ the vertices of $G_{2}$, then $W$.edges ()$\subseteq$ the edges of $G_{2}$. The theorem is a consequence of (63).

(66) Let us consider supergraphs $G_{1}, G_{2}$ of $G$ extended by vertex $v$ and edges between $v$ and $V$ of $G$. Then

(i) the vertices of $G_{1}=$ the vertices of $G_{2}$, and

(ii) every vertex of $G_{1}$ is a vertex of $G_{2}$.

Proof: The vertices of $G_{1}=$ the vertices of $G_{2}$.

(67) Let us consider supergraphs $G_{1}, G_{2}$ of $G$ extended by vertex $v$ and edges between $v$ and $V$ of $G$, and objects $v_{1}, e_{1}, v_{2}$. Suppose $e_{1}$ joins $v_{1}$ and $v_{2}$ in $G_{1}$. Then there exists an object $e_{2}$ such that $e_{2}$ joins $v_{1}$ and $v_{2}$ in $G_{2}$. 
(68) Let us consider supergraphs $G_{1}, G_{2}$ of $G$ extended by vertex $v$ and edges between $v$ and $V$ of $G$. Then there exists a function $f$ from the edges of $G_{1}$ into the edges of $G_{2}$ such that

(i) $f \uparrow($ the edges of $G)=\mathrm{id}_{\alpha}$, and

(ii) $f$ is one-to-one and onto, and

(iii) for every objects $v_{1}, e, v_{2}$ such that $e$ joins $v_{1}$ and $v_{2}$ in $G_{1}$ holds $f(e)$ joins $v_{1}$ and $v_{2}$ in $G_{2}$,

where $\alpha$ is the edges of $G$. The theorem is a consequence of (67), (47), and (51).

Let $G$ be a loopless graph. Let us consider $v$ and $V$. Observe that every supergraph of $G$ extended by vertex $v$ and edges between $v$ and $V$ of $G$ is loopless.

Let $G$ be a non-directed-multi graph. Let us note that every supergraph of $G$ extended by vertex $v$ and edges between $v$ and $V$ of $G$ is non-directed-multi.

Let $G$ be a non-multi graph. Note that every supergraph of $G$ extended by vertex $v$ and edges between $v$ and $V$ of $G$ is non-multi.

Let $G$ be a directed-simple graph. One can verify that every supergraph of $G$ extended by vertex $v$ and edges between $v$ and $V$ of $G$ is directed-simple.

Let $G$ be a simple graph. Let us observe that every supergraph of $G$ extended by vertex $v$ and edges between $v$ and $V$ of $G$ is simple.

Now we state the proposition:

(69) Let us consider a supergraph $G_{1}$ of $G_{2}$ extended by vertex $v$ and edges between $v$ and $V$ of $G_{2}$, a walk $W$ of $G_{1}$, and vertices $v_{1}, v_{2}$ of $G_{2}$. Suppose $V \subseteq$ the vertices of $G_{2}$ and $v \notin$ the vertices of $G_{2}$ and $W$.first ()$=v_{1}$ and $W$.last ()$=v_{2}$ and $v_{2} \notin G_{2}$.reachableFrom $\left(v_{1}\right)$. Then $v \in W$.vertices () . The theorem is a consequence of (64).

Let us consider $G_{2}, v, V$, and a supergraph $G_{1}$ of $G_{2}$ extended by vertex $v$ and edges between $v$ and $V$ of $G_{2}$. Now we state the propositions:

(70) Suppose $V \subseteq$ the vertices of $G_{2}$ and $v \notin$ the vertices of $G_{2}$ and $G_{2}$ is acyclic and for every component $G_{3}$ of $G_{2}$ and for every vertices $w_{1}, w_{2}$ of $G_{3}$ such that $w_{1}, w_{2} \in V$ holds $w_{1}=w_{2}$. Then $G_{1}$ is acyclic.

Proof: Consider $E$ being a set such that $\overline{\bar{V}}=\overline{\bar{E}}$ and $E$ misses the edges of $G_{2}$ and the edges of $G_{1}=$ (the edges of $\left.G_{2}\right) \cup E$ and for every object $v_{1}$ such that $v_{1} \in V$ there exists an object $e_{1}$ such that $e_{1} \in E$ and $e_{1}$ joins $v_{1}$ and $v$ in $G_{1}$ and for every object $e_{2}$ such that $e_{2}$ joins $v_{1}$ and $v$ in $G_{1}$ holds $e_{1}=e_{2}$. There exists no walk $W$ of $G_{1}$ such that $W$ is cycle-like.

(71) Suppose $V \subseteq$ the vertices of $G_{2}$ and $v \notin$ the vertices of $G_{2}$ and $\left(G_{2}\right.$ is not acyclic or there exists a component $G_{3}$ of $G_{2}$ and there exist vertices $w_{1}, w_{2}$ of $G_{3}$ such that $w_{1}, w_{2} \in V$ and $\left.w_{1} \neq w_{2}\right)$. Then $G_{1}$ is not acyclic. 
(72) Suppose $V \subseteq$ the vertices of $G_{2}$ and $v \notin$ the vertices of $G_{2}$ and for every component $G_{3}$ of $G_{2}$, there exists a vertex $w$ of $G_{3}$ such that $w \in V$. Then $G_{1}$ is connected.

Proof: For every vertex $u$ of $G_{1}$ such that $u \neq v$ there exists a walk $W_{1}$ of $G_{1}$ such that $W_{1}$ is walk from $u$ to $v$. For every vertices $u, w$ of $G_{1}$, there exists a walk $W_{1}$ of $G_{1}$ such that $W_{1}$ is walk from $u$ to $w$.

Let $G$ be a connected graph, $v$ be an object, and $V$ be a non empty set. Note that every supergraph of $G$ extended by vertex $v$ and edges between $v$ and $V$ of $G$ is connected.

Let us consider $G_{2}, v, V$, and a supergraph $G_{1}$ of $G_{2}$ extended by vertex $v$ and edges between $v$ and $V$ of $G_{2}$. Now we state the propositions:

(73) Suppose $V \subseteq$ the vertices of $G_{2}$ and $v \notin$ the vertices of $G_{2}$ and there exists a component $G_{3}$ of $G_{2}$ such that for every vertex $w$ of $G_{3}, w \notin V$. Then $G_{1}$ is not connected.

Proof: Consider $G_{3}$ being a component of $G_{2}$ such that for every vertex $w$ of $G_{3}, w \notin V$. Set $v_{1}=$ the vertex of $G_{3}$. There exists no walk $W$ of $G_{1}$ such that $W$ is walk from $v_{1}$ to $v$.

(74) Suppose $V \subseteq$ the vertices of $G_{2}$ and $v \notin$ the vertices of $G_{2}$ and there exists a component $G_{3}$ of $G_{2}$ such that the vertices of $G_{3}$ misses $V$. Then $G_{1}$ is not connected. The theorem is a consequence of (73).

Let $G$ be a non connected graph and $v$ be an object. One can check that every supergraph of $G$ extended by vertex $v$ and edges between $v$ and $\emptyset$ of $G$ is non connected.

(75) Let us consider a supergraph $G_{1}$ of $G_{2}$ extended by vertex $v$ and edges between $v$ and $V$ of $G_{2}$. Suppose $V \subseteq$ the vertices of $G_{2}$ and $v \notin$ the vertices of $G_{2}$. Then $G_{1}$ is complete if and only if $G_{2}$ is complete and $V=$ the vertices of $G_{2}$.

Proof: For every vertices $u, v$ of $G_{1}$ such that $u \neq v$ holds $u$ and $v$ are adjacent.

Let $G$ be a complete graph. Observe that every supergraph of $G$ extended by vertex the vertices of $G$ and edges between the vertices of $G$ and the vertices of $G$ is complete.

Now we state the propositions:

(76) Let us consider a supergraph $G_{1}$ of $G_{2}$ extended by vertex $v$ and edges between $v$ and $V$ of $G_{2}$. Suppose $V \subseteq$ the vertices of $G_{2}$ and $v \notin$ the vertices of $G_{2}$. Then

(i) $G_{1} \cdot \operatorname{order}()=G_{2} \cdot \operatorname{order}()+1$, and

(ii) $G_{1} \cdot \operatorname{size}()=G_{2} \cdot \operatorname{size}()+\overline{\bar{V}}$. 
(77) Let us consider a finite graph $G_{2}$, an object $v$, a set $V$, and a supergraph $G_{1}$ of $G_{2}$ extended by vertex $v$ and edges between $v$ and $V$ of $G_{2}$. Suppose $V \subseteq$ the vertices of $G_{2}$ and $v \notin$ the vertices of $G_{2}$. Then $G_{1}$.order ()$=$ $G_{2} \cdot \operatorname{order}()+1$.

(78) Let us consider a finite graph $G_{2}$, an object $v$, a finite set $V$, and a supergraph $G_{1}$ of $G_{2}$ extended by vertex $v$ and edges between $v$ and $V$ of $G_{2}$. Suppose $V \subseteq$ the vertices of $G_{2}$ and $v \notin$ the vertices of $G_{2}$. Then $G_{1} \cdot \operatorname{size}()=G_{2} \cdot \operatorname{size}()+\overline{\bar{V}}$.

Let $G$ be a finite graph, $v$ be an object, and $V$ be a set. One can verify that every supergraph of $G$ extended by vertex $v$ and edges between $v$ and $V$ of $G$ is finite.

\section{REFERENCES}

[1] Grzegorz Bancerek, Czesław Byliński, Adam Grabowski, Artur Korniłowicz, Roman Matuszewski, Adam Naumowicz, Karol Pąk, and Josef Urban. Mizar: State-of-the-art and beyond. In Manfred Kerber, Jacques Carette, Cezary Kaliszyk, Florian Rabe, and Volker Sorge, editors, Intelligent Computer Mathematics, volume 9150 of Lecture Notes in Computer Science, pages 261-279. Springer International Publishing, 2015. ISBN 978-3319-20614-1. doi:10.1007/978-3-319-20615-8_17.

[2] Grzegorz Bancerek, Czesław Byliński, Adam Grabowski, Artur Korniłowicz, Roman Matuszewski, Adam Naumowicz, and Karol Pąk. The role of the Mizar Mathematical Library for interactive proof development in Mizar. Journal of Automated Reasoning, 61(1):9-32, 2018. do1:10.1007/s10817-017-9440-6

[3] Lowell W. Beineke and Robin J. Wilson, editors. Selected Topics in Graph Theory. Academic Press, London, 1978. ISBN 0-12-086250-6.

[4] John Adrian Bondy and U. S. R. Murty. Graph Theory. Graduate Texts in Mathematics, 244. Springer, New York, 2008. ISBN 978-1-84628-969-9.

[5] Sebastian Koch. About supergraphs. Part I. Formalized Mathematics, 26(2):101-124, 2018. doi $10.2478 /$ forma-2018-0009

[6] Gilbert Lee. Walks in graphs Formalized Mathematics, 13(2):253-269, 2005.

[7] Piotr Rudnicki and Andrzej Trybulec. Abian's fixed point theorem. Formalized Mathematics, 6(3):335-338, 1997.

[8] Klaus Wagner. Graphentheorie. B.I-Hochschultaschenbücher; 248. Bibliograph. Inst., Mannheim, 1970. ISBN 3-411-00248-4.

[9] Robin James Wilson. Introduction to Graph Theory. Oliver \& Boyd, Edinburgh, 1972. ISBN 0-05-002534-1. 